\title{
AN INVESTIGATION INTO THE GEOMETRY OF SEYFERT GALAXIES
}

\author{
C. J. Clarke ${ }^{1}$, A. L. Kinney ${ }^{1,2,3}$ \& J. E. Pringle $\mathrm{e}^{1,2}$ \\ ${ }^{1}$ Institute of Astronomy, University of Cambridge, Mandingley Road, Cambridge, CB3 \\ 0HA, United Kingdom \\ ${ }^{2}$ Space Telescope Science Institute, 3700 San Martin Dr., Baltimore, MD 21218 \\ ${ }^{3}$ Physics and Astronomy Department, Johns Hopkins University, Homewood Campus, \\ Baltimore, MD 21218
}

\begin{abstract}
We present a new method for the statistical investigation into the distributions of the angle $\beta$ between the radio axis and the normal to the galactic disk for a sample of Seyfert galaxies. We discuss how further observations of the sample galaxies can strengthen the conclusions. Our data are consistent with the hypothesis that AGN jets are oriented randomly in space, independent of the position of the plane of the galaxy. By making the simple assumption that the Standard Model of AGN holds, with a universal opening angle of the thick torus of $\phi_{\mathrm{c}}$, we demonstrate a statistical method to obtain an estimate of $\phi_{\mathrm{c}}$. Our data are not consistent with the simple-minded idea that Seyfert 1s and Seyfert 2s are differentiated solely by whether or not our line of sight lies within some fixed angle of the jet axis. Our result is significant on the $2 \sigma$ level and can thus be considered only suggestive, not conclusive. A complete sample of Seyfert galaxies selected on an isotropic property is required to obtain a conclusive result.
\end{abstract}

\section{Introduction}

We concern ourselves here with the angle $\beta$ at which the central accretion disk of an active galactic nucleus (as evidenced by the direction of the central radio jet) lies relative to the host-galaxy disk, and with the angle $\phi$ which is the angle between the line of sight and the radio axis (see Figure 1 ).

In the standard picture of active galactic nuclei (Antonucci 1993), it is the angle $\phi$ which determines whether a galaxy appears to us as a Seyfert 1 or as a Seyfert 2. The central nucleus is surrounded by a physically thick circular torus of molecular gas which ensures that the very central regions of the accretion flow (the broad line region) are visible only when the line of sight and the central disk axis (assumed to align with the radio structure) lie within about $\phi_{\mathrm{c}} \sim 30^{\circ}$ of each other (Osterbrock and Shaw 1988). It is a central prediction of the model, in its simplest form, that values of $\phi$ for Seyfert 1s should be on average smaller than values of $\phi$ for Seyfert $2 \mathrm{~s}$. The estimate of $\phi_{\mathrm{c}} \sim 30^{\circ}$ given by Osterbrock and Shaw comes from an estimate of the relative numbers of Seyfert 1 and Seyfert 2 galaxies per unit volume of space. 
The angle $\beta$ serves as a link with the innermost workings of the active galaxy - either as the memory of the activity-provoking event, or as a diagnostic for the structure of the accretion disk. We develop a method for evaluating the most likely distribution of such angles based on an observed set of position angles as seen on the sky plane, and apply our method to a data set culled from the literature. This study is a precursor to a larger study based on complete, unbiased samples of spiral active galaxies selected on nearly isotropic properties.

The minimum components for activity in the nuclei of galaxies are a central black hole plus a fueling source which feeds material onto the black hole $(\mathrm{BH})$. The processes for bringing material close to the core (within the innermost 10pc) of a galaxy, either for the initial formation of the $\mathrm{BH}$ or to provide fuel to the $\mathrm{BH}$, are of two sorts - those that trigger the gas internal to the galaxy to collapse to the core, and those that trigger gas external to the galaxy to collapse to the core. Internal triggers include cooling flows, bars in galaxies, which funnel material to the core (Shlosman et al. 1989, Thronson et al. 1989), or flybys of companion galaxies (Byrd et al. 1986) which shock the ISM of the host galaxy into collapsing (perhaps aided by the formation of a bar). External triggers are mergers between galaxies which bring material to the core from outside the galaxy (Hernquist 1989, Disney et al. 1995).

Spiral galaxies have ample cold gas available to be shocked into collapse to the core by fly-bys or bars, so that internal triggers are viable possibilities for causing activity in spirals. But spirals have so much gas in the disk that the gas can mask or interfere with the evidence for a recent merger. Merger models show that the gas merging with the spiral galaxy at random angles dissipates the angular momentum that is not aligned with the galaxy angular momentum, and settles into the plane of the disk so as to be indistinguishable from the native gas (see Walker, Mihos, \& Hernquist 1996, and Mihos \& Hernquist 1995 and references therein).

AGN in spiral galaxies appear to show precious little correlation between the direction of inner jets/ionization cones and the spiral disk axis (Ulvestad \& Wilson 1984; Brindle et al. 1990; Baum, O’Dea, de Bruyn, \& Pedlar 1993; Schmitt et al. 1997). They also show a tendency for the broad line Seyfert 1s to point out of the galaxy disk while the narrow line Seyfert 2s point closer to the plane of the disk (Schmitt \& Kinney 1996). This finding may, however, not be due to the intrinsic distribution of relative position angle, but rather due to the manner in which the active galaxies are detected for inclusion into samples. Of particular interest is the zone of avoidance discovered by Schmitt, Kinney, Storchi-Bergmann, \& Antonucci 1997 who concluded that there is apparently a zone within a cone of half angle $20^{\circ}$ of the spiral disk axis within which no radio axis falls. The active nucleus apparently knows where the galaxy plane is and avoids close alignment with it. Given that fueling by both internal gas and by external gas favors co-alignment between AGN nucleus and galaxy disk, the observed mis-alignment is intriguing and requires a theoretical explanation. it might indicate, for example that recently suggested ideas about radiation induced warping of accretion disks (Pringle 1996, 1997; Maloney, Begelman \& Pringle 1996) come into play 
both to randomize the directionality and to produce the ionization cones.

In this paper we undertake a more direct statistical analysis than has been attempted hitherto in order to try to determine the distributions of the angle $\beta$ between the jet axis and the normal to the galaxy disk. The main aim of the paper is to demonstrate the feasibility of such an analysis, and to elucidate what further observations might be required (apart from simply acquiring a larger and more uniform sample) in order to strengthen the conclusions. In Section 2 we describe the data set that we use. In section 3 we set up a description of the geometry of the situation and present our statistical method for obtaining the $\beta$ distribution together with some preliminary results. In Section 4 we present the conclusions of the analysis and discuss both how further observations could be used to enhance the results and the possible implications for theoretical models.

\section{The Data Set}

We base our analysis on the data set collected by Schmitt et al. (1997, henceforth SKSA). The full list of objects is presented in Table 1 of that paper. The sample includes all Seyfert galaxies in the literature which have been resolved in high resolution radio maps and which show linear radio structure as defined by Ulvestad and Wilson (1984). The position angles of the extended radio structure were taken from the literature, while the position angles of the host galaxy major axis were all verified using the Digitized Sky Survey Plates and assuming that the galaxy disk is intrinsically circular. No galaxies in the sample lie within 10 degrees of edge on. Such objects are apparently difficult to detect as Seyfert galaxies, perhaps because in such a case the active component would be entirely absorbed by the host galaxy disk. Our method requires a measurement of both $i$, the galaxy inclination, and the radio position angle. Since 4 of the objects in the Schmitt et al. sample of 46 have no measurement for $i$, we include 42 objects in our analysis. Of these 14 are Seyfert 1s and 28 are Seyfert 2s.

When using a sample of objects assembled from the literature, one must always consider selection effects. SKSA show that the Seyfert 1s and Seyfert 2s in the sample have comparable $6 \mathrm{~cm}$ radio fluxes. Likewise, the Seyfert 1s and the Seyfert $2 \mathrm{~s}$ are hosted by galaxies of similar morphological type. However, Ulvestad and Wilson (1984) note that overall, Seyfert 1 galaxies which are resolved in the radio display shorter elongated radio structures than radio resolved Seyfert 2 galaxies. The standard model of AGN (Antonucci 1993) would interpret this as being due to the Seyfert 1 galaxies being those which observed within about 30 degrees of the torus axis in order that the broad line region can be observed. The face-on orientation would therefore tend to foreshorten the radio emission on the sky. But meanwhile, such foreshortening may cause Seyfert 1 galaxies to fall out of the sample, since the radio emission must be linear to be included in the sample. This is a sort of blind spot in the sample, but it should be a randomly distributed blind spot since the Seyfert 1s that are excluded can still have their radio jet extending from any angle out of the host galaxy (except for in the plane of the host galaxy - but those objects do not seem to make it into the sample in great numbers). The exclusion of Seyfert 1s with exactly face-on tori from 
the sample should affect absolute numbers of Seyfert 1's versus Seyfert 2s, but it should not affect the distributions of position angle overall. Thus this might be expected to affect the $\phi$ distributions for Seyfert 1s, but should have little impact on the $\beta$ distribution.

Radio flux and size generally correlate with far infrared power (Sanders et al. 1989). This sample contains the objects among the Seyfert galaxies which are detected and resolved in the radio. Thus these are also the brightest and most extended in the radio. The main selection effect is therefore the relatively benign one of selecting the top end of the luminosity function. The phenomenology of the most luminous of the Seyfert galaxies may differ from the phenomonlogy of the less luminous. An investigation of such luminosity effects could well be of interest, but will have to wait for deeper and higher resolution radio data.

We note that, although we are using the same sample as SKSA. our approach to the data is different. SKSA, in common with previous authors (for example, Ulvestad \& Wilson 1984, Baum et al. 1993) made use of just the distribution of position angle $\delta$ between the radio axis and the apparent galaxy major axis. Here we make use of the fact that for almost all of the sample we know the galaxy inclination (assuming that the galaxy disk is intrinsically circular) as well as $\delta$. This enables us to undertake a more direct approach by constraining the geometry of individual objects, as we describe below. To take this point further we show in Figure 2 the distributions of pairs $(\delta, i)$ for both the Seyfert 1s and the Seyfert 2s. Evident from the Figure the selection effect on inclination $i$, in that near face-on and near edge-on galaxies have been excluded form the sample. Using a KS test, the distributions in $i$ are not found to differ between the two samples, and indeed do not differ significantly from a uniform distribution over the sphere within the apparent sample limits. However the $\delta$ distributions of the Seyfert 1s and the Seyfert 2s do differ, with the probability that the two samples are drawn from the same distribution being less that 1 per cent. This difference can be seen clearly in the Figure, with the Seyfert 2s predominating in the range $0^{\circ}<\delta<30^{\circ}$.

\section{Statistical Analysis}

\subsection{Geometry}

For each galaxy we can determine two observational parameters, $i$ and $\delta$. The angle $i$ is the inclination of the plane of the galaxy to the plane of the sky, or equivalently the angle between the line of sight and the vector normal to the galaxy plane. The angle $i$ lies in the range $0^{\circ}<i<90^{\circ}$. We use a Cartesian coordinate system OXYZ (see Fig. 1, cf. Fig. 5 of SKSA) so that OX lies along the apparent major axis of the galaxy disk, OY lies along the apparent minor axis, and thus $\mathrm{OZ}$ is the vector normal to the galaxy plane. In these coodinates the unit vector in the direction of the line of sight is

$$
\mathbf{k}_{s}=(0,-\sin i, \cos i) .
$$

The angle $\delta$ corresponds to the position angle between the apparent major axis of the galaxy and the direction of the radio jet projected onto the plane of the sky. By convention $\delta$ is taken to lie in the range $0^{\circ}<\delta<90^{\circ}$. 
For a given value of $\delta$ and $i$, the direction of the jet, which we denote by a unit vector $\mathbf{k}_{j}$ is determined to lie on a great circle drawn on a unit sphere centred at the origin of our coordinate system. In the OXYZ coordinates described above the great circle is the set of points:

$$
\begin{gathered}
\mathbf{k}_{j}=\left(k_{j x}, k_{j y}, k_{j z}\right) \\
=(\cos \delta \sin \phi, \\
\sin \delta \cos i \sin \phi-\sin i \cos \phi, \\
\sin \delta \sin i \sin \phi+\cos i \cos \phi),
\end{gathered}
$$

where $\phi$ is the angle between the vectors $\mathbf{k}_{s}$ and $\mathbf{k}_{j}$ and formally lies in the range $-180^{\circ}<$ $\phi<180^{\circ}$. We should also note that there is a mirror symmetry to the problem about the apparent minor axis of the galaxy, that is about the OYZ plane. In terms of our coordinates this translates into the statement that reversing the direction of the OX axis leaves the problem unchanged. Thus, formally, the sign of $k_{j x}$ is not an observationally meaningful quantity, or in other words the jet vector in fact lies on one of two great circles which are reflections of each other in the OYZ plane. We have therefore simplified the discussion by considering just one of these great circles.

Of course not every point on the great circle is physically accessible for the reason that we assume that the observable jet is the one that lies above the disk plane (as seen from Earth). If we define $\beta$ as the angle the jet vector $\mathbf{k}_{j}$ makes with the disc normal OZ, then we see from Equation (3.1.2) that

$$
\begin{gathered}
\cos \beta=k_{j z} \\
=\sin \delta \sin i \sin \phi+\cos i \cos \phi .
\end{gathered}
$$

Thus the only relevant values of $\phi$ are those which give $0^{\circ}<\beta<90^{\circ}$, or $\cos \beta>0$. In terms of $\phi$, this means that $\phi$ lies in the range $\phi_{1}<\phi<\phi_{1}+180^{\circ}$, where $\phi_{1}$ is the value of

$$
\phi_{1}=\tan ^{-1}(-\cot i / \sin \delta),
$$

which lies in the range $-90^{\circ}<\phi_{1}<0^{\circ}$. We note that physically, if $\phi<0$, then the jet vector is projected against the half of the galaxy disk which is nearest to us, whereas $\phi>0$ corresponds to the jet being projected against the half of the galaxy disk which is furthest from us. From the current data we cannot discriminate between these two possibilities, but see, however, the discussion in Section 5.

\subsection{The minimum value of $\beta$}


We have seen that a given pair of values of $i$ and $\delta$ constrains the jet vector $\mathbf{k}_{j}$ to lie on a great circle $\mathbf{k}_{j}(\phi)$ on the unit sphere centred on the galaxy. The great circle passes through the line of sight and makes an angle $b$ with the plane of the galaxy (the $O X Y$ plane), where

$$
b=\cos ^{-1}(\cos \delta \sin i) .
$$

This means that we can set a lower limit to the value of $\beta$, which corresponds to the place where the great circle comes closest to the pole OZ. This value, which we denote by $\beta_{\min }$ is given by

$$
\beta_{\text {min }}=\cos ^{-1}\left(\sin ^{2} \delta \sin ^{2} i+\cos ^{2} i\right)^{1 / 2} .
$$

We note, however, that if we were able to distinguish between the near and far sides of the galaxy, that is between $+\phi$ and $-\phi$, then we would be able to decide which of the two segments of the great circle, on either side of $\phi=0$, the jet direction corresponds to. In that case we would have

$$
\begin{gathered}
\beta_{\text {min }}=\cos ^{-1}\left(\sin ^{2} \delta \sin ^{2} i+\cos ^{2} i\right)^{1 / 2}, \\
\quad \text { for } \phi>0 \\
=i, \text { for } \phi<0
\end{gathered}
$$

Furthermore, we also note that if we were able to determine if the jet is approaching us or receding from us (that is we were able to determine the sign of $\mathbf{k}_{j} \cdot \mathbf{k}_{s}=\cos \phi$ ), then this would further restrict the allowable range of $\phi$, (in particular to decide whether $\phi$ is greater or less than $90^{\circ}$ ) and so enable a more accurate determination of $\beta_{\text {min }}$.

However, for the purposes of this paper, in which the observational data does not tell us which side of the galaxy disk is closer to us, and in which we do not know whether the jet is approaching or receding, we must take $\beta_{\text {min }}$ as given by Equation (3.2.2). In Figure 3 we display the values of $\beta_{\text {min }}$ for the galaxies in our sample, distinguishing between the Seyfert $1 \mathrm{~s}$ and the Seyfert $2 \mathrm{~s}$.

We stress that while these results only give us one sided limits to $\beta$, the results themselves depend only on the geometry of the situation, and do not require any statistical modelling or additional assumptions. In this sense the conclusions drawn from them are robust and irrefutable. Thus we can state that, for example, more than 80 percent of the Sy2s in our sample have $\beta$ greater than $20^{\circ}$, more than 50 percent of them have $\beta$ greater than $30^{\circ}$, and more than 20 percent of them have $\beta$ more than $54^{\circ}$. For the Sy1s in our sample we can state that more than 80 per cent of them have $\beta$ greater than $17^{\circ}$, more than 50 percent of them have $\beta$ greater than $25^{\circ}$, and more than 20 per cent have $\beta$ more than $32^{\circ}$. These findings agree with the conclusions of Schmitt et al.(1997) who present evidence for a $20^{\circ}$ zone of avoidance around the galactic pole. 
It might be tempting to draw the conclusion from Fig. 2 that the distributions for $\beta_{\min }$ (or equivalently the distributions for the angles $b$ ) differ for the Seyfert 1 s and the Seyfert 2s. However, applying a KS test to the distributions puts the probability that the two sets of data are drawn from the same distribution as 29 percent. This is an indication that based on the $(\delta, i)$ pairings alone (Fig. 2) one should not expect to be able to find a significant difference in the values of $\beta$ for Sy1s and Sy2s.

\subsection{Estimation of the $\mathbf{P}(\beta)$ distribution}

Here we describe a method whereby, in principal, we may arrive at an estimate for the distribution of angles $\beta$ the jet vectors in our sample make with the galaxy disk normal. We denote this distribution in terms of a probability distribution $\mathrm{P}(\beta)$, which is defined so that:

$$
\int_{0}^{\pi / 2} P(\beta) d \beta=1
$$

Thus, for example if the jets axes were randomly orientated in space, and thus were independent of the orientation of the galaxy disk, then we would find that $\mathrm{P}(\beta)=\sin \beta$.

If we define the jet axis in terms of the angles $\beta$ and $\theta$ with respect to the coordinate axes OXYZ, so that

$$
\mathbf{k}_{j}=(\sin \beta \cos \theta, \sin \beta \sin \theta, \cos \beta),
$$

(see Fig. 1), then we note that the probability distribution $\mathrm{P}(\beta)$ corresponds to the full two-dimensional probability distribution $\mathrm{P}(\beta, \theta)$ integrated over the azimuthal angle $\theta$. In order to proceed further we need to make the assumption that the full two dimensional distribution $\mathrm{P}(\beta, \theta)$ is in fact independent of $\theta$. This assumption is a simple one and is valid provided that there are no selection effects which act preferentially to select in favour of jets with particular values of the azimuth $\theta$. Since the angle $\theta$ is defined in terms of coordinates which have a particular orientation relative to our line of sight, such a selection effect cannot be ruled out completely. Indeed in the Standard Model of AGN, in which the difference between Seyfert 1s and Seyfert 2s depends on whether $\phi$ is greater or less than $\phi_{\mathrm{c}}$, it is clear that this assumption is not a good one when applied to Seyfert 1s or Seyfert 2s separately. However, when applied to a complete sample of Seyferts as a whole (which we do not have), the assumption may indeed be a good one.

We begin by noting that if we were able to measure the actual value of $\beta$ for each galaxy jet, then again assuming that there are no significant selection effects, we could simply plot a histogram of $\mathrm{P}(\beta)$ directly from the observed sample. However we are unable to determine an exact value of $\beta$ for each galaxy in our sample, because we do not know where on the great circle the jet vector lies - that is we do not know the value of $\phi$ in Equation (3.1.2). We can recognize, however, that if we already knew $\mathrm{P}(\beta)$ (which we are of course attempting to determine) then we would be able to convert this information into a probability distribution for $\phi$, that is we would be able to determine the likelihood of the jet vector lying at various points along the great circle. This is the basis of the method which we employ. We also note 
that of course, as discussed above, if we knew in addition the sign of $\phi$ and/or the sign of $\mathbf{k}_{j} \cdot \mathbf{k}_{s}=\cos \phi$, then we could further restrict the position of the jet direction along the great circle defined by $i$ and $\delta$ (see Section 5).

We start by assuming the we know $\mathrm{P}(\beta)$, and in particular we start from the assumption that the jets are randomly orientated so that $\mathrm{P}(\beta)=\sin \beta$. We call this initial assumed distribution $P_{i n}(\beta)$. This implies that along a great circle, the assumed local surface (i.e. two-dimensional) probability density of jet orientations is $P_{i n}(\beta) / \sin \beta$. If the jet directions were randomly orientated, then the probability of finding a jet vector in a particular galaxy at a point on the unit circle between $\phi$ and $\phi+d \phi$ would be proportional to $|\sin \phi| d \phi$. The necessity for the factor $|\sin \phi|$ can be seen by noting that it enables the method to reproduce correctly a randomly orientated jet distribution. Thus for a given $P_{i n}(\beta)$ the probability of finding the actual jet between $\phi$ and $\phi+d \phi$ would be $\left[P_{i n}(\beta) / \sin \beta\right]|\sin \phi| d \phi$.

We now proceed by taking each data point and distributing it over the unit sphere according to the above formula derived from the assumed $P_{i n}(\beta)$. Having done that we may then integrate over azimuth on the sphere and obtain another estimate of the $\beta$ distribution, which we call $P_{\text {out }}(\beta)$. Clearly if $P_{\text {in }}$ and $P_{\text {out }}$ are the same (or do not differ significantly), then we have a good estimate of the real $\beta$ distribution. If they differ significantly, however, then we may, in principle, use the above method iteratively!.

Because we have only a small sample (even when both the Sy1s and the Sy2s are taken together) we simplify the method by binning the data. We bin the data in 5 bins equally spaced in $\cos \beta$, so that if the jets are randomly orientated we expect equal numbers of galaxies to fall into each bin. In our estimation procedure, we note that, since we redistribute each galaxy among the bins according to some probability distribution, the actual number of galaxies assigned statistically to each bin may not be a whole number. This in turn implies that the statistical analysis of the procedure is not straightforward. For simplicity, we adopt the use of $\sqrt{ } n$ error bars, where $n$ is the (not necessarily whole) number in each bin, We expect this to give us a reasonable estimate of the likely error due to sampling.

In Figure 4 we show the result obtained for $P_{\text {out }}(\beta)$, using the input assumption that $P_{i n}(\beta)$ corresponds to random jet orientations, or in other words, the input assumption is that each jet could lie anywhere randomly along the great circle defined by $i$ and $\delta$. As can be seen from the Figure, there is no evidence in the current sample that the jets orientations differ significantly from being uniformly distributed. We note that this result does not contradict the findings of Schmitt et al. (1997) who concluded, in line with our findings in Section 3.2, that there is a significant underabundance of AGN with $\beta<20^{\circ}$. Our choice of bin size as shown in Figure 2 means that the first bin corresponds to $0^{\circ}<\beta<37^{\circ}$, which results in such an effect being washed out in the analysis.

\subsection{The Seyfert 1 and Seyfert 2 Subsamples}

\footnotetext{
${ }^{1}$ We note briefly that the simple iterative procedure as stated here is not well posed, and that an extra constraint such as an assumption about the smoothness of the $\beta$ distribution, or its closeness to being uniform, is likely to be required.
} 
In all of the above we have not differentiated between the AGN according to whether they are Seyfert 1s or Seyfert 2s. However, if we are prepared to make the hypothesis that the Standard Model of AGN is correct, then we can make further statistical tests. We shall, for illustration, take the strong hypothesis that AGN are either Seyfert 1s or Seyfert 2 s depending on whether $\phi$ is less than or is greater than (respectively) some canonical, and universal, value $\phi_{\mathrm{c}}$. Given this assumption we may make an estimate for the $P(\beta)$ distribution for the Seyfert 1s and Seyfert 2s separately. For the Seyfert 1s we assume that each jet can be anywhere along the great circle, with the proviso that $\phi<\phi_{\mathrm{c}}$. Similarly for the Seyfert $2 \mathrm{~s}$ we make the same assumption except that now we require $\phi>\phi_{\mathrm{c}}$.

However, using the assumption that the value of $\phi$ is the only difference between the Seyfert 1s and the Seyfert 2s, it follows that the $P(\beta)$ distributions we obtain for the two subsamples should be the same. This means that if we vary the assumed value of $\phi_{\mathrm{c}}$ until the difference between the two $P(\beta)$ so obtained is a minimum, then we obtain an estimate of the value of $\phi_{\mathrm{c}}$. We have carried out this calculation, using only 3 bins in $\cos (\beta)$, since the Seyfert 1 sample only contains 14 entries. We use a simple $\chi^{2}$ test (with two degrees of freedom) to see if the two distributions differ. We find that the difference between the two distributions is a minimum at $\phi_{\mathrm{c}} \simeq 40^{\circ}$, but that the goodness of fit is poor, with the probability that the two distributions are different being 96 per cent (i.e approximately $2 \sigma$ ). We show the 'best fit' in Figure 5. This implies that the simple hypothesis we have made in order to determine an estimate for $\phi_{\mathrm{c}}$ is not sustained. It may be that $\phi_{\mathrm{c}}$ is not a universal constant, for example it could be a function of $\beta$, or the opening of the torus might not be cylindrically symmetric. Alternatively, it might be that the $\beta$ distributions for Sy1 and Sy2 galaxies are significantly different. A possible hint in this direction comes from the observation that for the 'best fit' (and indeed the fits for all values of $\phi_{\mathrm{c}}$ ) the Seyfert 1 distribution is more concentrated towards low values of $\beta$ than is the case for Seyfert 2s. Since this difference between the Sy1 and Sy2 distributions is not apparent in the $\beta_{\min }$ distributions (Section 3.2) it seems that it is the assumption that the Sy1s and Sy2s are differentiated in terms of $\phi$, an assumption which is fundamental to the Standard Model, which drives the suggestion of the difference in the $\beta$ distributions.

Given the lack of systematic selection of the sample, the small sample size, and the $2 \sigma$ level of significance of the result, this result can only be called suggestive and not conclusive.

\section{Conclusions and Discussion}

We have investigated the angle $\beta$ which the jet axis $\mathbf{k}_{j}$ makes with the normal to the galaxy disk plane. By considering the minimum value of $\beta$ which is allowed by the data for each galaxy, we are able to conclude (Section 3.2) that for both Sy1s and Sy2s most of then have angles $\beta$ which are greater than $25^{\circ}-30^{\circ}$. This is a straightforward conclusion, independent of theoretical models, and implies not only that jets perpendicular to the disk plane are not the norm, but also that strict alignment is avoided.

Because the data are all derived from a projection of a three-dimensional situation onto the two-dimensional plane of the sky, we cannot expect to be able to reconstruct the full three- 
dimensional picture without resorting to some statistical modelling. We have demonstrated how such modelling can be carried out (Section 3.3) with a minimum of assumptions. Our estimate of the $\beta$ distribution for the sample as a whole (Fig. 4) shows that the distribution is consistent with the distribution which would result from the jet being pointed randomly with respect to the galaxy.

By making the further hypothesis that $\phi_{\mathrm{c}}$ is a universal constant, we have been able to estimate a value for $\phi_{\mathrm{c}}$, by choosing that value which makes the deduced $\beta$ distributions for the Sy1s and the Sy2s most consistent with each other. We obtain the value $\phi_{\mathrm{c}} \simeq 40^{\circ}$, but note that the two distributions so obtained for $P(\beta)$ are significantly different. Thus simple-minded application of the Standard Model appears to lead to a contradiction. The assumption that Sy1s and Sy2s are differentiated in terms of the angle $\phi$ seems to result in general in a Sy1 $\beta$ distribution which displays significantly smaller values of $\beta$ than the Sy 2 distribution. Using a simple $\chi^{2}$ test to evaluate the significance of this result, the hypothesis that the Sy1 and the Sy2 distributions, $P(\beta)$, are the same is ruled out at the 4 per cent level, or approximately $2 \sigma$. Given the lack of systematic selection criteria of the sample, and the fact that similarity is ruled out only to $2 \sigma$, this result is not conclusive but only suggestive. A complete sample, selected on an isotropic property, is required to obtain a conclusive result.

Thus, we have established the feasibility of this kind of analysis, which provides observational measures of the $\beta$ distribution and provides some hope that the assumptions underlying the Standard Model for AGN might be subject to observational scrutiny. It is evident that a larger sample would be of value. With a large enough sample, it will also be necessary to take proper account of measurement errors in the angles $i$ and $\delta$, and also to have a better understanding of the selection effects present in the sample. One example of such an effect is the probability that small values of $\phi$ are likely to be ruled out of the sample, because severe foreshortening of the jet means that it is impossible to measure $\delta$ with any precision. Even without a larger sample, however, further constraints on the geometry obtained by further observations of the sample galaxies would strengthen the conclusions.

One such constraint would be to determine which side of the minor axis lies physically closer to us. If we can achieve this, we can remove the degeneracy in our knowledge of the sign of $\phi$ and in effect improve the statistics in the sample by about a factor of two since we can constrain the jet to lying along only a particular segment of the great circle. One method by which we can discover which is the nearer edge of the galaxy is by taking long slit spectroscopy along both the major and the minor axis. Along the minor axis we would look for dust effects and thus need the bluest possible waveband, where the dust has the most effect. By differencing the spectrum of the top minor axis from that of the bottom minor axis we should be able to distinguish the foreground, less-reddened side from the background, more reddened side. Along the major axis we would look for velocity differences, and thus need spectral regions with distinct galactic spectral features, which requires slightly redder spectral regions. Then, along with the assumption that the spiral arms are always trailing, the velocities along extreme ends of the major axis, or the farthest ends intercepted by a 
spiral arm, would easily allow us to distinguish front from back. The differences in velocity that we will need to detect will be of order $600 \mathrm{~km} / \mathrm{s}$ independent of the inclination $i$. By placing the long slit on both the major and minor axes, we determine foreground side versus background side with two independent methods. Both methods are more accurate for edgeon than for face-on galaxies, but similarly, the answer is more constraining for the edge-on than for the face-on case.

A further, though more difficult, method of constraining the geometry would be to attempt to determine whether the radio jet is moving towards us as opposed to away from us (not to be confused with whether the radio jet is on the front side of the galaxy as opposed to the back side of of the galaxy). This would then limit the sign of $\cos \phi$ and so improve our statistics by further limiting the arc on the great circle along which the jet can lie. Determining whether the jet is into the sky plane or out of the sky plane is not easy. For some of the stronger, more highly polarized jets, it may be possible to look for depolarization along the length of the jet. This method is very effective for the grand radio jets, as demonstrated by Laing (1988) and Garrington et al. (1988) but may be problematic for the smaller jets found in Seyfert galaxies. As a polarized jet is observed further into its own local halo, the halo serves to depolarize the jet, thus showing which end of the jet is observed through a higher column density.

Finally we note that although further observations, both in terms of a larger sample, and in terms of further observations of the current sample, will provide better constraints, it is clear that we have already demonstrated the power of this kind of approach. Even the preliminary results which we present here, based on simple observations of an initial sample, provide interesting implications for theoretical interpretation of Seyfert galaxies. In particular it may be necessary to provide a theoretical explanations (a) for why the inner accretion disks (as evidenced by the jets) appear to have little knowledge of the plane of the galaxy and (b) for why either there is little difference in the $\phi$ values for Sy1s and Sy2s or the inner disks in Sy1s are more aligned with the galaxy plane than the disks in Sy2s. It will be of interest to see whether these results hold up with the acquisition of further and better observational data.

\section{Acknowledgements}

We would like to thank Ski Antonucci and Henrique Schmitt for discussions and critical comments to the manuscript. We would like to thank Rupert Knight for his late arrival, which allowed C.J.C. to complete calculations before becoming otherwise engaged. ALK would like to thank the Institute of Astronomy for hospitality during a sabbatical visit.

\section{References}

Antonucci, R., 1993, ARA\&A, 31, 473

Baum, S.A., O’Dea, C.P., de Bruyn, A.G., \& Pedlar, A., 1993, ApJ, 419, 553

Brindle, C., Hough, J. H., Bailey, J. A., Axon, D. J., Ward, M. J., Sparks, W. B. \& McLean, I. S. 1990, MNRAS, 244, 577 
Byrd, G.G., Valtonen, M.J., Sundelius, B., \& Valtaoja, 1986, AA, 166, 75

Disney, M.J., Boyce, P.J., Blades, J.C., Boksenberg, A., Crane, P. Dehargeng, J.M., Macchetto, F., Mackay, C.D., Sparks, W.B., \& Phillipps, S., 1995, Nature, 376, 150

Garrington, S.T., Leahy, J.P., Conway, R.G., and Laing, R.A. 1988, Nature 331, 147

Hernquist, L., 1989, Nature, 340, 687

Laing, R.A. 1988, Nature, 331, 149

Maloney, P.R., Begelman, M.C. \& Pringle, J.E., 1996, ApJ, 472, 582

Mihos, J.C., \& Hernquist, L. 1996, ApJ, 448, 41

Osterbrock, D.E., \& Shaw, R.A. 1988, ApJ, 327, 89

Pringle, J.E., 1996, MNRAS, 281, 357

Pringle, J.E., 1997, MNRAS, in press

Sanders, D.B., Phinney, E.S., Neugebauer, G., Soifer, B.T., and Mathews. K. 1989, ApJ 347,29

Schmitt, H.R. \& Kinney, A.L., 1996, ApJ, 463, 498

Schmitt, H.R., Kinney, A.L., Storchi-Bergmann, T., \& Antonucci R., 1997, ApJ, 477, 623 (SKSA)

Shlosman, I., Frank, J., Begelman, M.C. 1989, Nature, 338, 45

Thronson, H.A., Hereld, M., Majewski, S., Greenhouse, M., Johnson, P., Spillar, E., Woodward, C.E., Harper,D.A., \& Rauscher, B.J., 1989, ApJ, 343, 158

Ulvestad J.S. and Wilson A.S., 1984 ApJ 285, 439.

Walker, I.R., Mihols, J.C., \& Hernquist, L. 1995, ApJ, 460, 121

\section{Figure Captions}

Figure 1. The plane of the galaxy is the $\mathrm{X}, \mathrm{Y}$ plane, placed so that the apparent major axis of the galaxy is the $\mathrm{X}$ axis. The line of sight, $Z^{\prime}$, thus lies in the plane of the paper, and $i$ is the angle of inclination of the galaxy. The radio jet is represented by the arrow (and referred to in the text as $\mathbf{k}_{\mathbf{j}}$ ). The angle at which the central accretion disk axis lies relative to the host galaxy disk is given by $\beta$. The angle between the line of sight and the radio axis is $\phi$. In the Standard Model, active galaxies with $\phi$ less than some canonical value of about $30^{\circ}$ are observed as Seyfert 1 galaxies, while active galaxies with $\phi$ greater than that value are observed to be Seyfert 2 galaxies. The position angle between the radio axis and the apparent major axis of the galaxy is given by $\delta$. Note that the two observable angles are $i$ and $\delta$. The azimuthal angle of the radio jet is given by $\theta$.

Figure 2. The difference in position angle on the sky (dpa) between the jet and the apparent major axis of the galaxy (also referred to in the text as $\delta$ ) is plotted against the inclination angle $i$ of the galaxy plane (with $i=90^{\circ}$ corresponding to an edge-on galaxy). The Seyfert $1 \mathrm{~s}$ are shown as filled squares and the Seyfert $2 \mathrm{~s}$ as crosses.

Figure 3. The cumulative distributions of $\beta_{\text {min }}$ are shown for both the Syls and the Sy2s. The distributions are scaled so that the total number of each subsample appear as a percentage. The Seyfert 1s are shown as filled squares and the Seyfert 2s as crosses. 
Figure 4. The estimated $P(\beta)$ distribution obtained for the whole sample (Sy1s and Sy2s). The bins are chosen to have equal sizes in $1-\cos (\beta)$ so that a uniform distribution on the sky would give equal numbers in each bin (as shown by the horizontal line). The error bars in each bin have been simply estimated as $\sqrt{ } n$ where $n$ is the number assigned to the bin. Figure 5. The estimates of the $P(\beta)$ distributions for (a) the Sy1s and (b) the Sy2s which result from the assumption that the Sy1s and Sy2s are differentiated only in terms of whether $\phi$ is less than, or greater than, the 'best fit' value of $\phi_{\mathrm{c}} \simeq 40^{\circ}$. As for Figure 4 the bin sizes are equal in $1-\cos (\beta)$. 


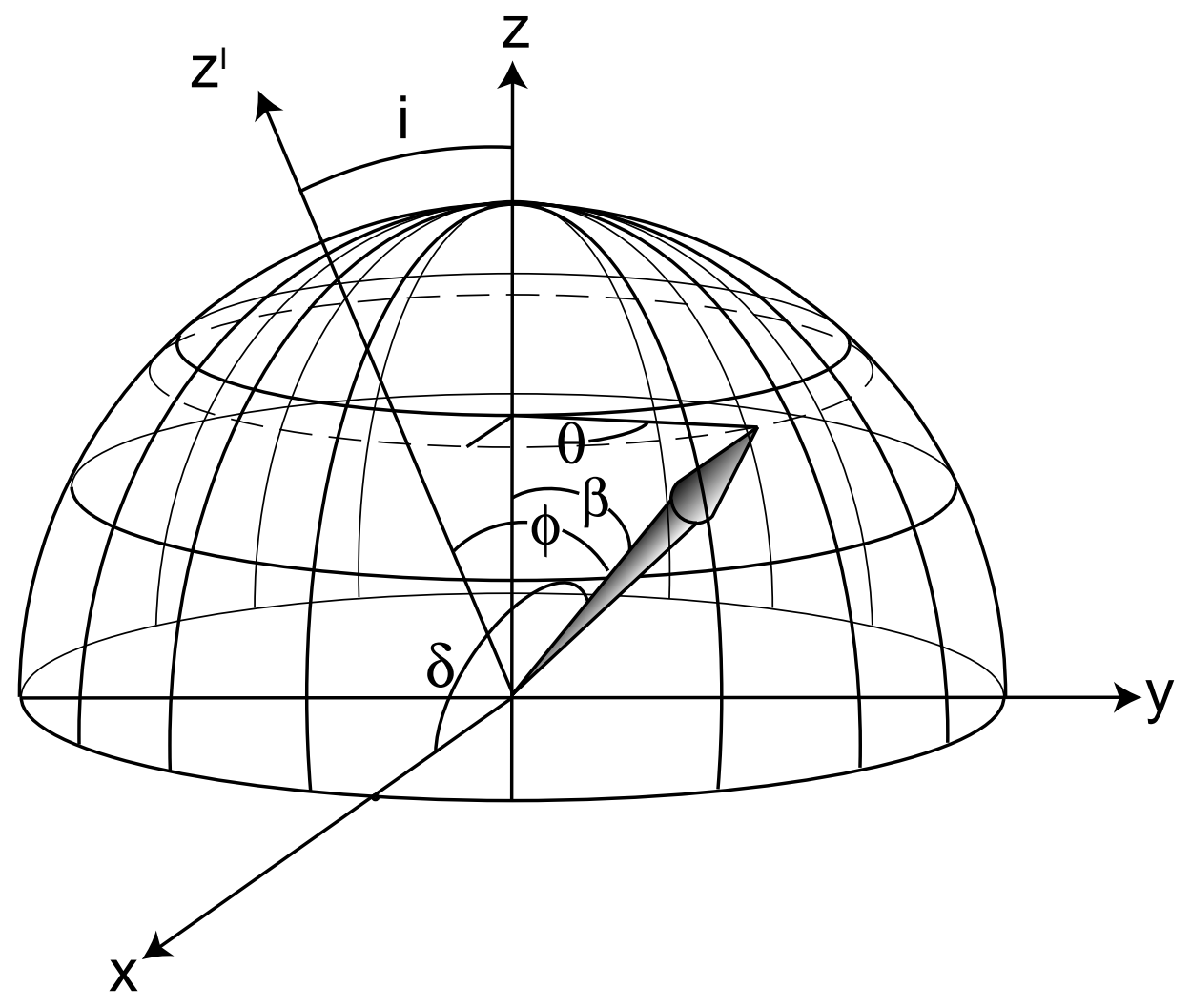




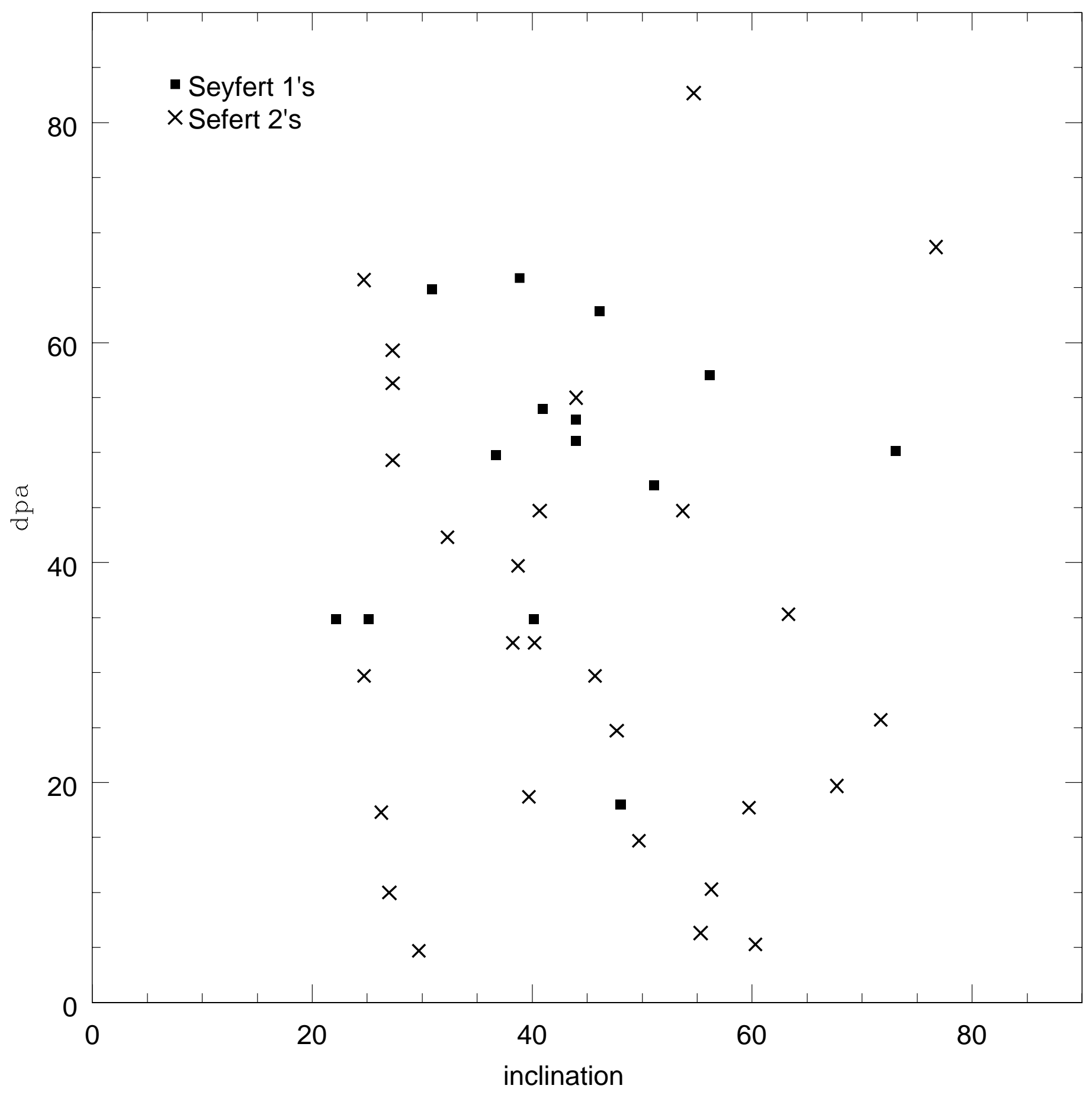




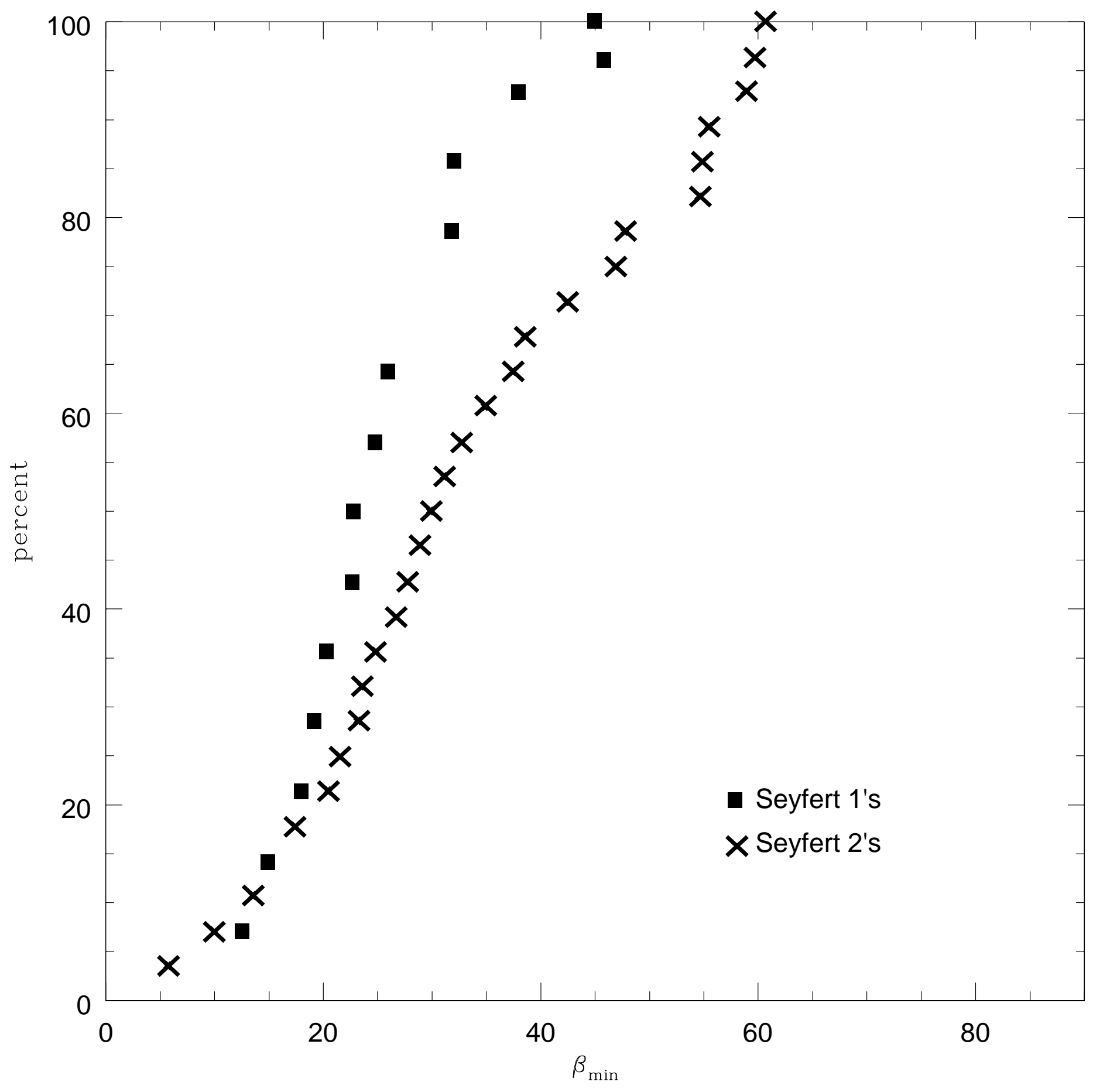




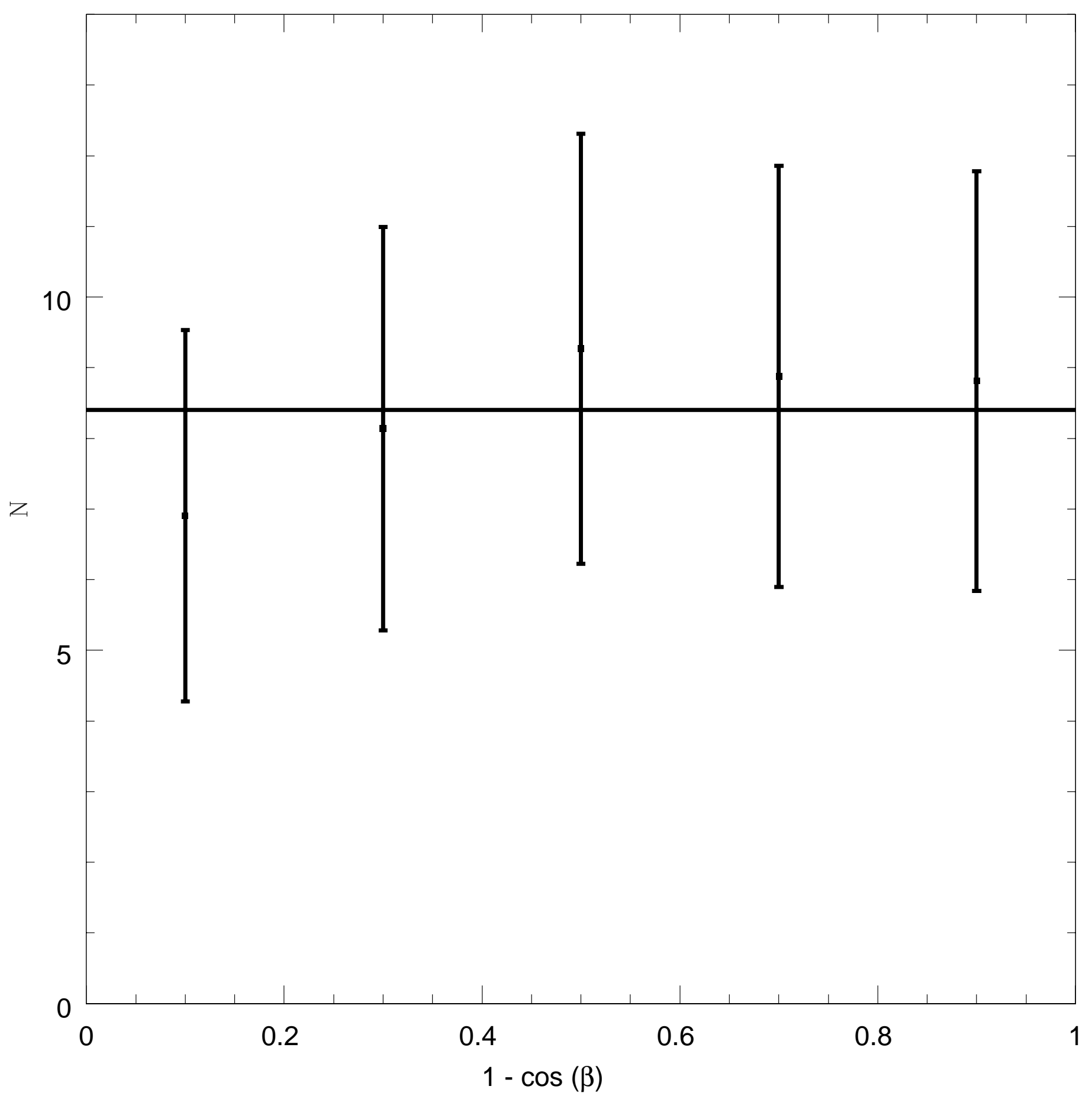




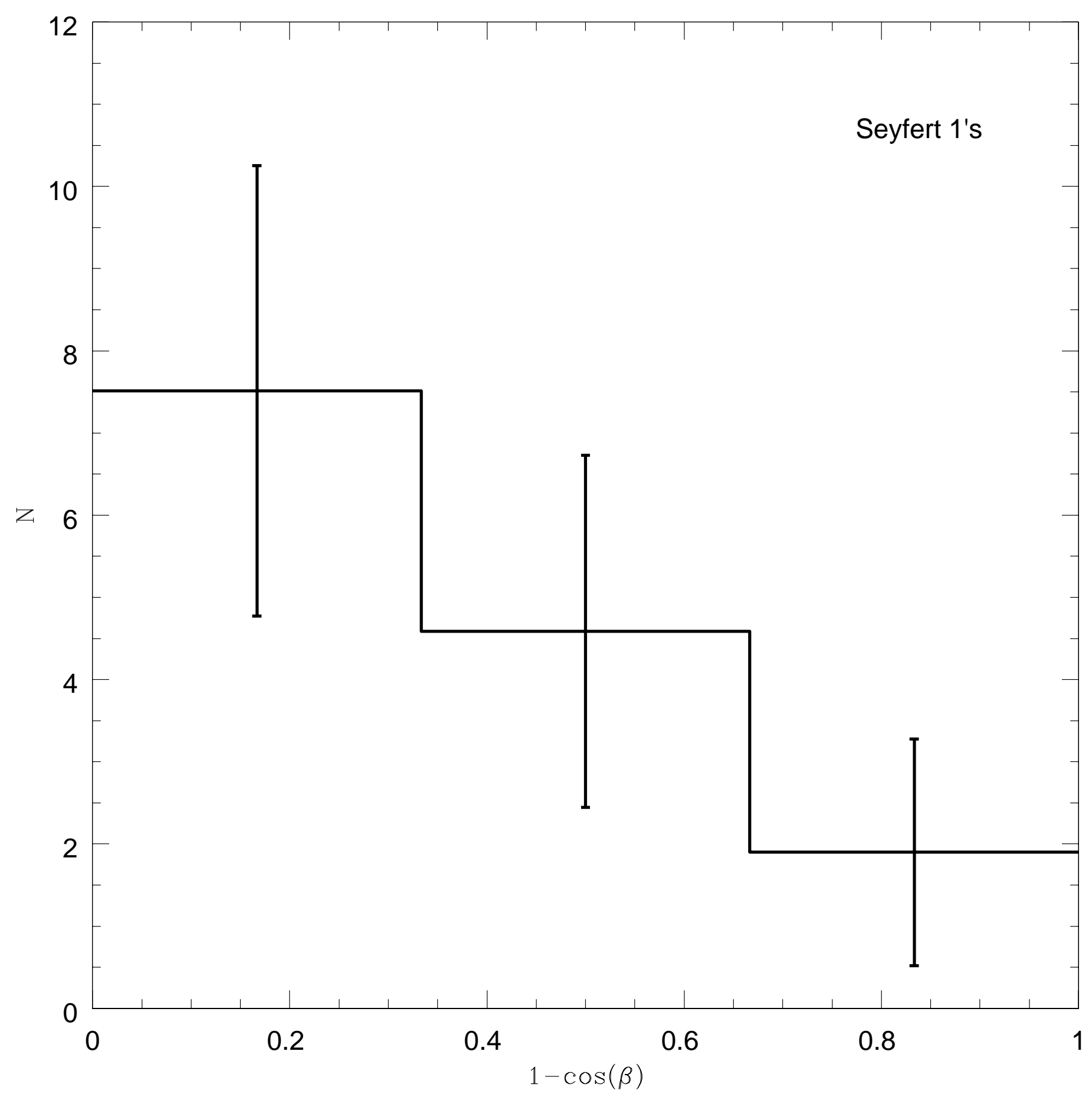




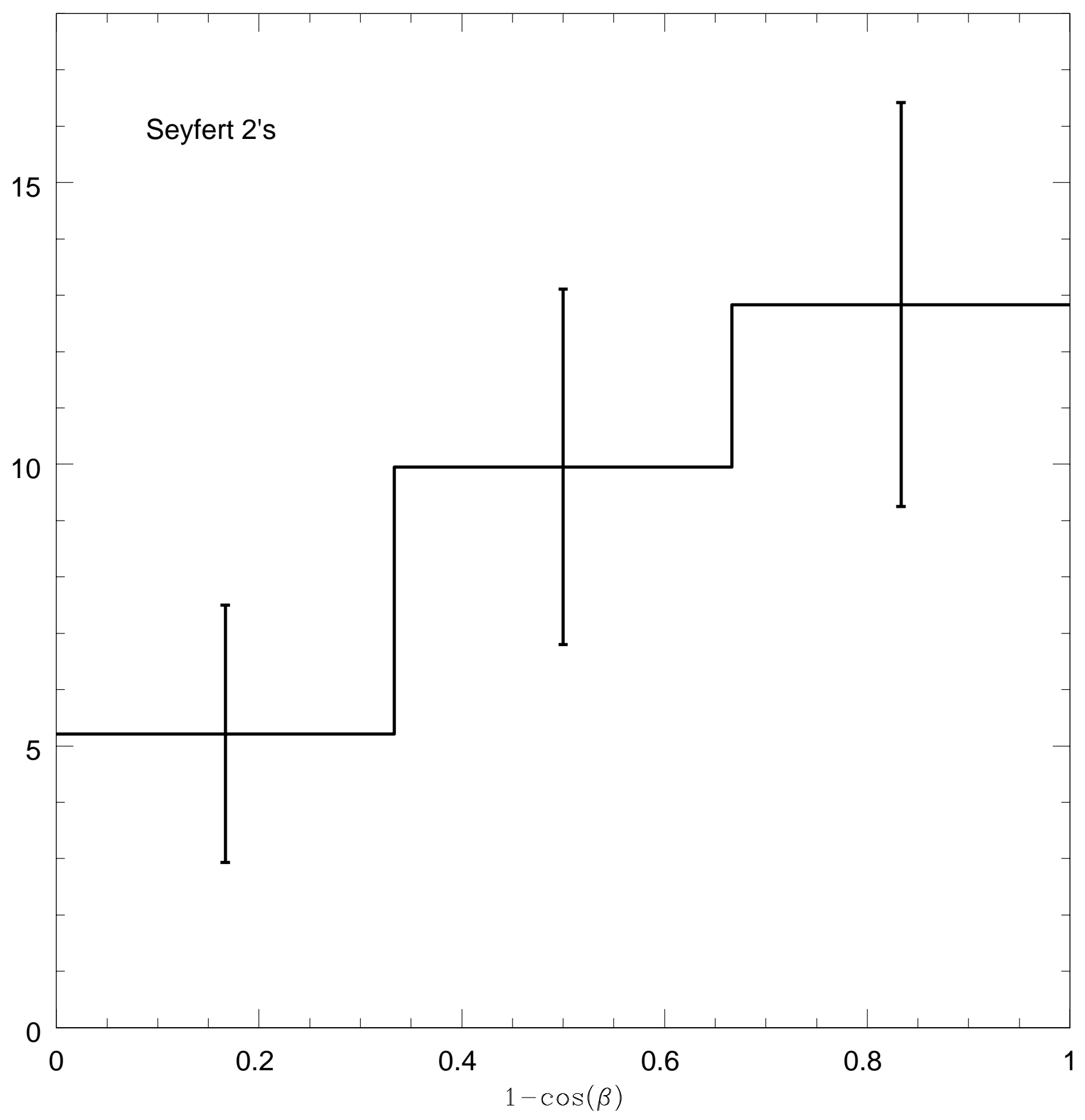

